\title{
Construction Robot Development Using Genetic Algorithm Technique
}

\author{
Anna M. McCrea*, Dr. Ronie Navon** \\ *Senior Lecturer - School of Construction, Faculty of Built Environment, South Bank \\ University, Wandsworth Rd., London SW8 2JZ, UK. \\ Researcher - Robotics Unit, Department of Civil Engineering, City University. \\ Northampton Square, London EC1V OHB, UK. \\ mccreaaa@vax.sbu.ac.uk \\ **Feld Academic Senior Lecturer - Technion, Department of Civil Engineering and \\ National Building Research Institute, Technion City, 32000 Haifa, Israel. \\ cvronie@tx.technion.ac.il. Internet: http://techunix.technion.ac.il/ cvronie/
}

\begin{abstract}
In this paper, it has been shown how to utilize the genetic algorithm (GA) technique in construction robot development. The investigation of the problem is carried out on the example of an automated device for the restoration of steel bridges, as the technology of the process poses a high level of health hazard and causes environmental pollution. Using GA as an instrument, allows to optimize the robot's key parameters simultaneously, based on the criteria of collision avoidance, percentage of coverage, productivity and dexterity. After formulating of the problem, the choice of the optimization parameters is justified and the procedure for analysis of the inter-relation between the task characteristics and the robot design is set out. Then, the optimization tool (GA) is introduced as the technique yielding optimal values for the parameters. Separating the problem into two-step approach follows, with full analysis and justification. The illustration of the optimization model uses simulation and computer graphics as a tool. Discussion on the effectiveness of the method and the quality of the expected outcome summarizes the paper.
\end{abstract}

\section{INTRODUCTION}

The objective of selecting the most effective robot for a designated range of activities is aiming at obtaining optimal values of certain robot parameters, based on chosen criteria.

This paper demonstrates how to quickly and efficiently utilize, combine and modify already well developed optimization technique and how to implement it on a practical application, achieving results ready to be used on a particular range of tasks. Because the project is orientated towards a specific application, it is expected that it would yield superior results than the domain independent analysis. This approach is developed and tested on the 'model task' (restoration work on steel bridges) by selecting and optimizing the parameters of an automated device to be used for that purpose. 


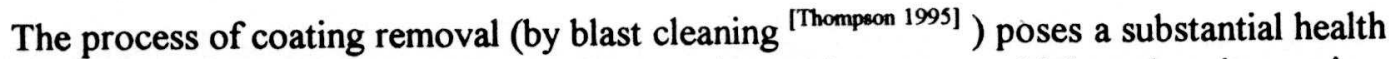
hazard and causes high levels of pollution to the environment, which makes it a prime candidate task for automation. Bridges being structures of varied geometry and dimensions have always been considered difficult and expensive to access for maintenance. Therefore, selection of a flexible, lightweight manipulator for tool delivery and handling, and further integration of other components, such as mobile carriage and access platform, could produce an automated device which would improve health and safety record and reduce pollution.

\section{PROBLEM FORMULATION OF ROBOT PARAMETERS OPTIMIZATION}

A logical approach to the optimal robot selection, is to configure and design the robot (main configuration and the wrist), that optimally carries out a specific range of tasks. The aim of this research is to develop a tool and the methodology for optimizing the values of selected parameters of the bridge restoration robot based on selected criteria.

The selected parameters for optimization are: (i) main configuration, namely the type of the first 3 DOFs; (ii) wrist configuration; (iii) link lengths; (iv) joint working ranges; (v) joint velocities and accelerations.

The above are optimized based on the following criteria: (i) collision avoidance; (ii) percentage of coverage; (iii) dexterity and (iv) productivity, which although grouped together, represent different levels of significance. Collision avoidance has to be always ensured, as this determines the ability to deliver the task at all. Dexterity determines the ability to reach required locations with specified tool orientation in the predetermined trajectory. In other words it indicates the regions of the working envelope not 'properly' reached due to insufficient orientation flexibility of the tool. Productivity being a very complex criterion, cannot be fully addressed, without extensive dynamic assessment of the robot, here an initial, simplified kinematic profile-based approach is assumed. The percentage of coverage indirectly indicates the amount of manual labour required to complete the task.

The parameters listed earlier also require further expansion. The first two parameters deal with the type of robot joints, of which only two basic types are commonly used - they are either revolute ( $R$ - exhibit rotary motion about an axis) or prismatic ( $P$ - exhibit linear motion along an axis). The axes of the first three joints cater for the delivery and location of the tool, therefore these are also referred to as major axes; the axes of the remaining joints, referred to as the minor axes or a wrist, establish the orientation of the tool. The geometry of the work envelope is mainly determined by the sequence of joints used for the first three axes. The dexterity (the ability to deliver the tool in the required orientation) of the envelope is governed by the wrist configuration.

Simultaneous assessment and optimization of several parameters can only be justified if they show interdependency. In this context interdependency is displayed as mutual influence, as shown in the following examples: changing the type of configuration implies different types of motors with different working ranges and motion characteristics. Altering the link lengths within the same configuration results in changes in the working ranges of joints in order to maintain the size of the working envelope. A need for increased joint motion (velocity and acceleration) results in different kinds of motors and therefore influences the working ranges of joints, or even their type. Changes to the wrist configuration, being mainly responsible for tool orientation, produce the requirement for altering the joint working ranges and link lengths in order to maintain the ability to deliver a specifically oriented tool to the workspace. 


\section{GENETIC ALGORITHMS IN OPTIMIZATION}

The Genetic Algorithm (GA) is a class of stochastic search algorithms which are based on ideas borrowed from natural evolution and biogenetics. Possible solutions are formed as strings of parameters with each parameter being represented as a gene, which is constrained by minimum and maximum values. Each 'candidate solution' is then represented by a string of genes - 'a chromosome' - or a set of such strings. A randomly created number of these solutions form a set of candidate solutions (population) which undergoes a constant transformation subjected to genetic operations such as reproduction, crossover or mutation (to be explained below) and being ranked for effectiveness (fitness to survive). New generations of chromosomes are formed by randomly selecting pairs of chromosomes 'parents' - and swapping part of their genes to form 'child' chromosomes (crossover), with a selection of parents being biased to more effective (fit) subsequent parents. Occasionally a randomly selected gene in a limited number of chromosomes (offsprings) is mutated, which helps to generate unexpected directions in the solution space and allows new genes or building blocks, which do not appear in neither parent, to be created. The value of each candidate is referred to as its 'fitness', based on the adopted evaluation method. Better individuals should be assigned larger fitness scores and the evaluation function should execute quickly. Some degree of randomness is allowed in the generation of offspring to allow the weaker building blocks to have a chance to survive.

The measure of success is the convergence of the population, meaning all the members of the population becoming identical. However, convergence should not be accepted without conscious assessment, as the population could very often converge on a sub-optimal solution.

Once a GA is designed and ready to run, the implementator is faced with the task of selecting appropriate control parameters. Proper choice of these control parameters is necessary to maximize the probability that the GA will produce good results.

The following values for these parameters were chosen (for the computation), based on the conclusions of [Grefenstette, 1992] : (i) GA's crossover probability - 0.55; (ii) GA's mutation probability -0.002 ; (iii) population size - 100 . Although the preferable approach would be to enable the GA to modify its own parameters dynamically during the search, but the number of evaluations which can be performed in a reasonable amount of time would not allow the GA enough evaluations to modify its search techniques to any significant degree ${ }^{\text {[Roston, 1994] }}$.

Robotic systems are known to benefit greatly if the individual sub-systems, such as mechanical structure, control circuitry, trajectory specifications, etc., are optimized [Davidor, ${ }^{19901}$. Several aspects of robotic design and programming have been addressed using GAs. Problems such as: mobile manipulator path planning ${ }^{[\mathrm{ZhaO}, \text { et al, 1993] }}$, robot motion planning [Ahuactzin, et al, 1991] , design of redundant manipulators [Davidor, et al, 1991] found their approach justification in using genetic based techniques.

\section{UTILIZATION OF GA IN ROBOT PARAMETERS OPTIMIZATION}

Genetic Algorithms are proven [Goldberg, ${ }^{1989]}$ to be an excellent technique for exploring large search spaces for optimal or near optimal solutions, as explained in the previous section. In robot selection and design the technique also showed promising results [Davidor, 1990] Selection of the robot begins with the optimization of its basic configuration (first three major axes) based on the required range of tool locations, determined by the bridge geometry and its 
mode of operation. However, representing a wide range of robot configurations for GA based optimization is a very complex problem. Consequently, at this stage of the research, only a simplified version of this problem is considered for further analysis.

As the gross working envelope of a robot is defined by the locus of points in three dimensional space that can be reached by the end effector, the representation of the robot is based on this approach. The vector of joint variables and robot geometry, using direct kinematic calculations, determines the position and orientation of the end-effector. If the vector of parameters specified above is compiled in GA applicable format, every chromosome represents a point in 3-D space, which the robot is capable of reaching. Therefore, a string of chromosomes can geometrically describe several series of such points, or otherwise paths or trajectories, within the envelope. Assessment of how close these points are to the required predetermined end-effector locations yields preferable values of the robotic parameters, based on the criteria of percentage of coverage.

Task completion time (leading to productivity assessment) depends on the type of control over the motion of a robot with the optimal control incurring severe real-time computational load. On top of the computational load, mechanical constraints such as torque, speed and/or acceleration of gears, harmonic drives, etc. place physical limitations on the robot's speed. Thus with the optimization of the total time control not being stressed in the robot design, alternative approach to productivity is taken on board. Coordinated motion (i.e., simultaneous movement of several manipulator axes in order to arrive at the desired endpoint) of all axes is assumed. The data including the angles of rotational movement of each joint, corresponding speeds and velocities allows to calculate the time required to rotate each joint (assuming the trapezoidal velocity profile). The longest time indicates the approximate overall time required to move the manipulator.

The required trajectory's geometry, orientation and location is a direct product of the geometrical analysis of the workspace (here, the geometry of a selected part of the bridge) and assessment of the automation potential and operating conditions of the task tools (here, the maneuvering, position and orientation of the blasting nozzle).

Theoretically it is possible to represent all the optimization parameters in a single chromosome, however performing genetic operations on such a long representation and then quality or 'fitness' assessment of each string in the population, for each generation, lies outside the computing capabilities of today. Consequently the optimization process is divided here into two stages, at the first stage the robot configuration (first 3 DOFs), the length of the links and the joints' motion ranges are optimized. At the second stage, the wrist configuration and the joints' speed and acceleration are added to the optimal configuration determined at the first stage. It was possible to divide the process into two stages without violating the simultaneity principle as will be explained below.

\subsection{FIRST STAGE OPTIMIZATION}

Initially, the dimensions of the arm links and the joints' working sectors of the major axes are concentrated on. The working sectors of the joints refer to the reachable ranges in joint movement despite the physical limitations. The orientation of the tool (minor axes) is not taken into account at this stage, as the task of the coating stripping and the tool operational requirements are not too sensitive to minimal changes in the orientation. The parameters for optimization at this stage are, therefore: (i) 2 configuration types - R(z)R(y)R(y) (a robot with the following axes: first - revolute around the vertical axis $(\mathrm{z})$, the second and the third - 
also revolute but with their axis perpendicular to the plane of the paper $(y)), R(z) R(y) P(z)$ (a robot with the main configuration as follows: first two joints as for previous configuration with the third joint being prismatic with its slider along vertical axis (z)); (ii) the relationship between 2 link lengths, while keeping the robot's reach constant $\left(\mathrm{L}_{1}+\mathrm{L}_{2}=\right.$ constant); and (iii) joints' working ranges. The choice of the two configurations for this example was governed by the nature of the restoration task and the form and context of the transformation matrices, so that the crossover would not violate the logical structure of the chromosome.

The parameters at this stage are optimized, based on the criteria of collision avoidance and percentage of coverage. These two criteria are mainly influenced by the values of the parameters selected at this stage, so splitting the process into two stages did not violate the merit of simultaneity. Collision avoidance, as referred-to in this approach, means that any part of the robot does not intersect at any point with the bridge, which in this example depends on the location of the tool, and not its orientation. Collision avoidance is the criterion of primary importance. Percentage of coverage specifies the range of the assigned task which the robot can do, thus indirectly it indicates the amount of manual involvement in task completion and, again, is a function of location rather than orientation. Therefore, combining these two criteria uses the same representation. The two stage approach does not violate the simultaneity of the process, as the values of the parameters from the first stage are refined in the second one, and 'full' optimization of additional parameters is performed at the second stage.

Each chromosome represents indirectly a point in 3-D through a single set of joint variables (configuration). A range (string) of configurations describes the sequence of tool locations (using direct kinematics), which is the followed trajectory. The closer the representation approaches the planned path (trajectory) on a bridge surface, the higher its fitness. Optimization here is based on minimizing the distance between the trajectory followed by the model (representation) and the path on the bridge, and the ability to closely follow a number of trajectories located in various parts of the workspace.

In this approach the following notation (see Fig. 3) was adopted: $\mathbf{T}_{\mathbf{3 i}}(\mathbf{x}, \mathbf{y}, \mathbf{z}$ point coordinates) $(\mathrm{i}=1, \ldots, \mathrm{NxM})$ is a matrix specifying $\mathrm{M}$ trajectories of $\mathrm{N}$ points each, represented by the series of locations of the tool with respect to the base coordinate frame. $\mathbf{E}_{3 i}$ $(i=1, \ldots, N x M)$ is a similar matrix, but specifying the locations of the elbow while following the path, also with respect to a global frame. The number of paths $(M)$ can be investigated simultaneously, to check the ability to perform various tasks without relocation. $\mathbf{P}_{\mathbf{3 i}}$ $(i=1, \ldots, N x M$ ) specifies $\mathrm{N}$ number of "ideal" points to be reached in each of $\mathrm{M}$ paths in the workspace, marked in relation to the same reference frame. A fitness value $f_{1}$ is represented as the sum of the squared distances between $\mathbf{T}_{\mathbf{3 i}}$ and $\mathbf{P}_{\mathbf{3 i}}$ (to avoid offsetting by negative values). The minimum value of $\mathbf{f}_{1}$ indicates the best ability to reach all the points along the trajectories and awards the candidate the highest fitness.

At the same time evaluation of collision avoidance is inbuilt into the fitness function. The bridge geometry under analysis, is represented as an obstacle with its contour marked as serious of points $\mathbf{C}_{3 \mathbf{k}}(\mathrm{k}=1, \ldots, \mathrm{K})$, with $\mathrm{K}$ being number of points along the geometry contour. The intersection between any point along the elbow $\left(\mathbf{T}_{\mathbf{3 i}}-\mathbf{E}_{\mathbf{3 i}}\right)$ and the surface of the obstacle adds high penalty factor to $\mathbf{f}_{\mathbf{1}}$ for a candidate.

\subsection{SECOND STAGE OPTIMIZATION}

The optimization here, uses all the parameters from the previous stage, except that it includes only the 'fitter' configuration. The formerly assessed parameters: link lengths and 
first three joints' working ranges are refined at this stage, additional parameters: (i) two wrist configurations, (ii) minor axes joint working ranges, (iii) all joint velocities and accelerations are added. The criteria by which they are selected include: (i) dexterity and (ii) productivity.

The choice of wrist configurations was governed by their applicability and versatility, and concentrated on RPY (roll, pitch, yaw) and RPR (an Euler wrist). The evaluation of the wrist type arises from compatibility of the orientation of the standard orthonormal bases of all points along the trajectory, measured in relation to the base coordinate frame and the orientation of the orthonormal basis of the wrist (tool attachment point) calculated in relation to the same coordinate frame. Wrist's orientation is assessed analyzing rotational part of the transformation matrices within kinematic calculations. The unit vectors of the orthonormal bases (already oriented in the tool/task compatible position) of the points along the required trajectory $\mathbf{P}_{\mathbf{3 i}}$ are projected onto the orthonormal basis of the robot's base frame. The $3 \times 3$ matrix $\mathbf{R}_{\mathrm{ij}}$ represents the transformation matrix from the coordinates of $\mathbf{P}_{\mathbf{3 i}}$ with the respect to the global frame to the coordinates with respect to the frame in the robot's base frame. The rotational part of the final transformation matrix of the robot full 6 DOF configuration $\mathbf{A}_{\mathbf{i j}}$ already projects the unit vectors of the wrist onto the same, robot's base frame. Compatibility of the equivalent column vectors between the matrices results in matching orientation.

The evaluation of each representation with respect to productivity is based on the choice of the longest time needed to rotate each of the axes within each representation and then selecting the shortest times out of all the previously calculated longest ones within the population. The trapezoidal velocity profile is assumed for the calculations.

\section{APPROACH TO REPRESENTATION AND EVALUATION IN STAGE ONE}

The first stage parameters are encoded as a single chromosome (Fig. 1) consisting of: (i) the configuration type; (ii) link length combination and (iii) joint range, in a binary representation. The chromosome is built as $\mathrm{M}$ number of the sequences (trajectories) of $\mathrm{N}$ points in each. The number of paths $(\mathrm{M})$ and their location and orientation describe the level of versatility, or otherwise ability to get close to the boundaries of the workstation within the bridge geometry. The selection of paths would emerge from geometric analysis of the working environment (characteristics of the selected area in the bridge geometry).

The continuous ranges of parameters are divided into sets of discrete values, as continuous variables are difficult for GAs to handle. To solve the problem the range is divided into 64 (including 0) discrete cutoff values, numbers 0 to 63 serve as a type of shorthand for each specific numerical cutoff value. For the joint ranges, the search space is discretized and represented by 3 integers (one for each joint), each belonging to $(0, \ldots, 63)$ in a binary representation. Boundaries of working ranges are assessed, based on the working environment dimensions and practical motor considerations (here, initially $360 \mathrm{deg}$ ).

1 gene(config. type) $\mid 1$ gene (link length) $\mid$

3 genes $=$ chromosome 1 (point $N=1$ in $3 D \in$ path $M=1) \mid 3$ genes $=$ chrom. $2($ point $N=2$ in $3 D \in$ path $M=1)$

$\mid 3$ genes $=$ chrom. 3 (point $N=3$ in $3 D \in$ path $M=1) \mid$

3 genes = chrom. 4 (point $N=1$ in $3 D \in$ path $M=2) \mid 3$ genes = chrom. 5 (point $N=2$ in $3 D \in$ path $M=2) \mid$

3 genes $=$ chrom 6 (point $\mathrm{N}=3$ in $3 \mathrm{D} \in$ path $\mathrm{M}=2)$

Figure 1. First Stage String = Representation

13th ISARC 
Arriving to the optimal solution means in practice convergence to the representation of greatest fitness. This generates the boundaries of working ranges of first three joints.

The search space is sized as follows: first digit in a string is specifying configuration, link length is represented by 6 digits, joint angle range is represented by 3 times 6 digits. The length of the second link is set automatically due to the requirement from the arm to be of constant length. Each chromosome is encoded by 18 bits. The first digit specifying the configuration is located in the beginning of the string, as the type of configuration has to be consistent throughout the whole of each trial, similarly the link length has to be constant (the same robot geometry). $\mathrm{M}=2$ trajectories are analyzed, each consisting of $\mathrm{N}=3$ points, therefore the overall length of the string is $1+6+6 \times 18=115$ bits.

\subsection{EVALUATION}

It is important to make the evaluation process execute as quickly as possible because of the large number of times it is performed. GAs use the fitness value of each string of the current generation to decide if and how many copies of the string should be passed to the next generation. The fitness value can never become negative.

The evaluation at the first stage is based on the direct kinematic calculations, providing series of positions of end effector relative to the base frame. Fitness is assigned to each member of the population (each string), based on a combination of ability to follow closely the pre-set number and type of paths and collision avoidance. Distance between path points and tool position is calculated between the nearest points irrespective of their sequence, therefore a short routine of distance assessment has to be introduced, prior to the distance calculations. The closer the set of configurations brings the tool to the desired goal without collision, the better its rank will be. At the first stage of GA optimization, a fitness function is defined (Eqn. 1) as follows:

$\mathrm{f}_{1}=\sum_{i=1}^{N x M} \frac{1}{\left(T_{3 i}-P_{3 i}\right)^{2}+I}$

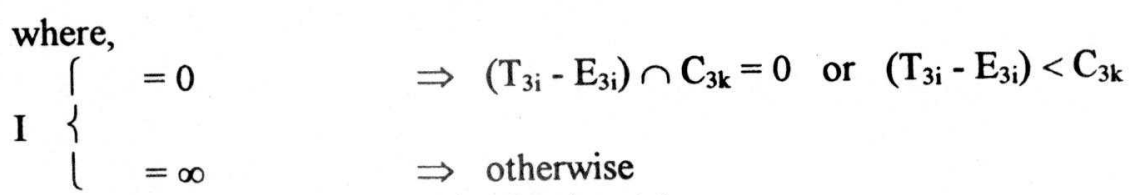

The variables are explained in Section 4.1.

\section{APPROACH TO REPRESENTATION AND EVALUATION IN STAGE TWO}

Essentially similar coding scheme to represent the parameters into genetic strings is assumed, but a different approach to the criterion assessment appears in the second stage. The previously coarse intervals in the ranges of the parameters for the first three major axes, become finer and partition the region within the boundary values where now the diversity is concentrated. Additionally, the three minor axes (wrist) are added to the representation, with their parameters solution space of a low resolution. Two minor (wrist) configurations chosen for the detailed analysis are: RPY and RPR, as described previously. Also all joints' accelerations and velocities are incorporated into the representation, at a low resolution as well. 
1 gene (link length) $\mid 1$ gene (wrist config.) $\mid 3 \times 3$ genes = chromosomes 1-3 (location of 3 points $\mathrm{N}=1$...3 in $3 D \in$ path $M=1 \mid 3 \times 3$ genes = chromosomes $4-6$ (orientation of 3 points $N=1 \ldots 3$ in $3 D \in$ path $M=1 \mid$

6 genes $=$ chrom .7 (joint velocities) $\mid 6$ genes $=$ chrom 8 (joint accelerations)

Figure 2. Second Stage String = Representation

The type of encoded representation at this stage consists of $\mathrm{N}$ times the following: 6 digit string for link length, one digit for manipulator type, 3 times 7 digits (127 divisions of the reduced working range) for the first three joints of the 'fitter' configuration (determined at stage one), 3 times 6 digits for manipulator joints' ranges, 6 times 6 digits for joint velocities and 6 times 6 digits for joint accelerations. $\mathrm{N}$ represents the number of points in a trajectory (here $N=3$ ). The string's length has therefore $6+1+3 \times\{3 \times 7+3 \times 6+6 \times 6+6 \times 6\}$ bits, which amounts to 340 bits. Three points in the trajectory have to belong to one of two paths from the previous stage, in order to generate credible results. Ideally the second criterion would be evaluated for the same number and size of the paths as at the first stage and only due to unrealistic time of generating results, it has to be reduced.

\subsection{EVALUATION}

At this stage, to evaluate the best individuals in the population, two criteria: (i) dexterity and (ii) productivity have to be addressed. The time of every attempt to reach each out of NxM points will be attributed to the longest time $t_{i}$ required to move each out of all six axes. The representation with the smallest sum value of the previously calculated longest times receives highest fitness. The distance to the points is not an objective, as they are selected from $\mathbf{P}_{3 \mathbf{i}}$ at the first stage. Trapezoidal velocity profile is specified for the motion. Part of fitness function relating to productivity (time) in this stage is assumed as follows (Eqn. 2a):

$\mathrm{f}_{2 \mathrm{a}}=\sum_{i=1}^{N \times M} \frac{1}{t_{i}}$

A separate, second component of the fitness function addresses the dexterity. The rotational parts of the transformation matrices are assessed for the final orientation of the wrist. This orientation is displayed through the final transformation matrix $\mathbf{A}_{\mathbf{i j}}$, where the column vectors represent the projection of the coordinates of the unit vectors of the wrist onto robot's base frame. All points along the path $\mathbf{P}_{3 \mathrm{i}}$ must also have allocated unit orthonormal vectors representing the required tool orientation. The columns of the matrix $\mathbf{R}_{\mathbf{i j}}$ indicate projection of the coordinates of the path point's unit vector onto the robot's base frame. The representation of the greatest fitness will have the maximum value of the squared reciprocal of the differences between the relevant column vectors of both matrices.

$\mathrm{f}_{2 \mathrm{~b}}=\cdot \sum_{\substack{i=1 \\ j=1}}^{N \times M} \frac{1}{\left(A_{i j}-R_{i j}\right)^{2}}$

The total evaluation function will consists of two components (Eqn.3):

$f_{2}=A x f_{2 a}+B x f_{2 b}$

A and B are 'weighting' factors assigned, depending on a relative importance of productivity against dexterity. 
At both stages the preferable solutions will receive numerically smaller fitness values, therefore a scheme needs to be employed which changes these small numerical values into higher fitness and employing sum of reciprocals seems to produce satisfactory results.

\section{GRAPHICAL REPRESENTATION OF THE ROBOT IN THE WORKPLACE}

The graphical description of the working environment is presented, in order to further clarify the workplace and the robot.

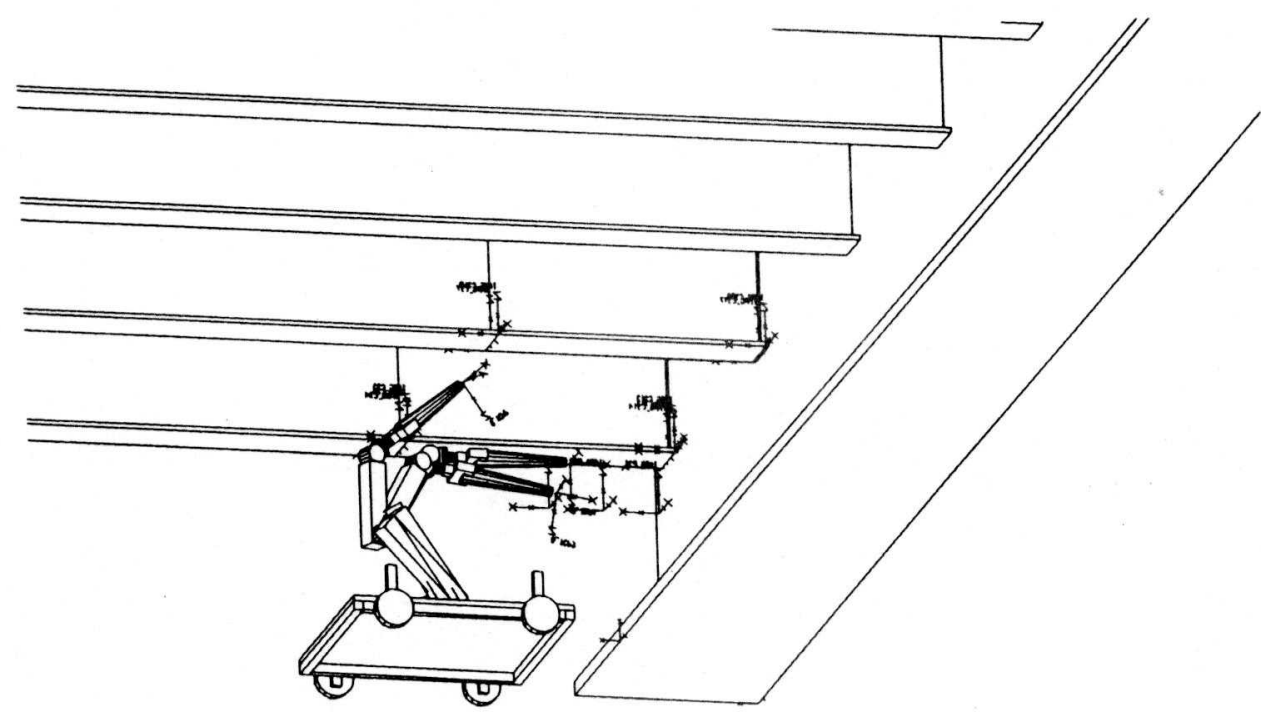

Figure 3. Illustration of the Optimization Model

The above figure visualizes an example of a part of a steel bridge with composite decking under restoration and representation of an RRR arm on a mobile platform positioned under the bridge. The global access platform, suspended from the bridge and supporting the robot, is omitted for clarity. On the bridge geometry, the thickened line joining all the 3-D points represents the contour of the obstacle $\left(\mathbf{C}_{3 k}\right)$ within the workspace. Two series of three points (one below the cross girder and one between the girders) mark two required paths for the tool end effector.

On the robot itself, the tool end effector is marked as $\mathbf{G}-\mathbf{T C P}_{\mathbf{3 i}}$ and Figure 3 shows three positions of the arm, hence three vectors $\mathbf{T}_{3 \mathrm{i}}$ and hence three point path followed by the tool. Consequently vectors $\mathbf{E}_{\mathbf{3 i}}$ display the path followed simultaneously by the elbow.

It will remain only to secure no intersection between any point along the arm and the contour of the obstacle to address collision avoidance. Furthermore, minimizing the distance between relevant points within $\mathbf{P}_{3 \mathbf{i}}$ and $\mathbf{T}_{3 \mathrm{i}}$ will generate the 'ideal' value of such parameters as joint working ranges, link lengths, type of the first 3 DOFs and the wrist configuration. 


\section{SUMMARY AND DISCUSSION}

Prior to any computations, a manual run of the representation was carried out to preliminary assess the potential outcome. The result, although without significance on its own, was within expected boundaries.

Introducing two stages into the optimization due to computing limitations, is not without logical implications. The optimal values of parameters assessed for the criteria of maximum percentage of coverage and collision avoidance, may not necessarily comply with optimal values of the same parameters optimized for productivity or dexterity. A solution to this problem is introduced in the second stage., the cutoff values of all the parameters were either oscillating around the optimal values from the first stage, at a fine resolution, or lie within optimal boundaries resulting from first stage.

Introducing the GA technique into robot design can generate more economical solutions, with the more appropriate types of motors and geometry more suited to the task. The dexterity criterion which in reality significantly reduces the size of the working envelope can be numerically evaluated and updated throughout the process, at every 'trial'.

\section{REFERENCES}

- Ahuactzin J.M., Talbi E-G., Bessiere P., Mazer E., (1991) Using Genetic Algorithms for Robot Motion Planning, Geometric Reasoning for Perception and Action, Workshop, Springer-Verlag, SLNC-708.

- Davidor Y.,Goldberg Y., (1989) An Evolution Standing on the Design of Redundant Manipulators, Parallel Problem Solving from Nature, 1st Workshop, Springer-Verlag, SLNC-496.

- Davidor Y., (1990) Genetic Algorithms and Robotics, World Scientific.

- Goldberg D.A., (1989) Genetic Algorithms in Search, Optimization and Machine Learning, Addison-Wesley Publishing Company.

- Grefenstette J.J., (1992) Optimization of Control Parameters for Genetic Algorithms, IEEE Computer Society Press.

- Roston G.P., (1994) A Genetic Methodology for Configuration Design, Ph.D. Thesis, Carnegie Mellon University.

- Thompson T.,Haas C.,Dailey Ch.,Traver A., (1995) An Assessment of Automated Surface Finishing Technologies, Proceedings of the Construction Congress of ASCE in San Diego.

- Zhao M., Ansari N. and Hou E., (1994) Mobile Manipulator Path Planning by a Genetic Algorithm, Journal of Robotic Systems 11(3):143-153. 